\title{
Energy Efficient Routing Protocol for Delay Tolerant Network Based on Fuzzy Logic and Ant Colony
}

\author{
Mohamed Ababou, Mostafa Bellafkih and Rachid El kouch \\ National Institute of Posts and Telecommunications, INPT, RAI2S, Rabat, Morocco \\ E-mail: \{ababou, bellafkih, elkouch\}@inpt.ac.ma
}

Received: 20 April 2017; Accepted: 06 July 2017; Published: 08 January 2018

\begin{abstract}
The messages routing in a DTN network is a complicated challenge, due on the one hand of intermittent connection between the nodes, the lack of the end-to-end path between source / destination and on the other hand, the constraints related to the capacity of the buffer and the battery. To ensure messages delivery in such an environment, the proposed routing protocols use multiple copies of each message in order to increase the delivery ratio. Most of these routing protocols do not take into account the remaining energy of nodes and the history on the relays that have already received a copy of the message in order to select the nodes that will participate in the message routing. This paper proposes a new approach named EERPFAnt inspired by the ant colony intelligence and improved by the fuzzy logic technique to select the best relay by combining the energy level of the nodes, as well as the information on the relay that have already received a copy of the message to estimate intelligently, the energy level of the nodes at the time of encounter with the desired destination. Simulation results will show that the proposed approach performances are better than those of Epidemic routing protocols, Spray and Wait and ProPHET.
\end{abstract}

Index Terms-DTN, Epidemic, Energy, ProPHET, Spray and Wait, Routing Protocol.

\section{INTRODUCTION}

Delay tolerant network (DTN) [1-3] is a network composed of mobile wireless nodes that exchange messages dynamically among them based on the store and forward mechanism. Each node in this network acts as a relay that participates in message routing, hop by hop, between the source and the destination of the message. However, DTN nodes have a limited capacity of the battery and the buffer. In addition, the high mobility of nodes in the network makes communication difficult from end to end between nodes.

The characteristics of DTN environments make traditional routing protocols [4] inadequate in this type of environment. To ensure the messages delivery in this environment, several approaches have been proposed, such as the routing protocols; Epidemic [5-7], Spray and Wait $[8,9]$ and ProPHET $[10,11]$.

Epidemic uses a blind strategy to route messages with the objective of minimizing the delivery delay. The use of an unlimited number of copies may generate a very large overhead and can cause in some cases the saturation of buffer nodes. Moreover, it depletes the nodes battery by wasting energy due to the transmission of unnecessary messages copies.

Spray and wait controls the number of copies forwarded by the Epidemic routing protocol by limiting the number of copies to be transmitted of each message in advance by the protocol settings, in order to fill the gaps of Epidemic routing protocol by reducing the amount of resources needed to deliver a message, while trying to maintain a good performance, but the disadvantage of this protocol is the random selection of nodes that will carry the copies in the spray phase, because it may be in some cases that the holders who have received a copy of the original message created by the source never meet the final destination.

ProPHET uses information on encounters between the nodes and the transitivity of contacts to calculate the probability of issue. However, it has the disadvantage of increasing the number of copies of messages disseminated in the network significantly. In addition, they don't take into account the energy consumption nor the messages already stored in the buffers of relays before deciding to choose those that will participate in message routing.

Previous work [12, 13] proposed a technique called Supp-trans, to improve performance routing protocols; Epidemic and Spray and Wait using information on the paths of messages that have been delivered to their final destination and analyzes the impact of mobility models on the proposed strategy.

This paper presents a new approach named EERPFAnt and evaluates it using simulations compared to the routing Protocols: Epidemic, Spray and Wait and ProPHET.

EERPFAnt is based on fuzzy logic [14] and the ant colony optimization [15] to propose a routing protocol for DTN network, which allows nodes to choose intelligently the relays that have the great chance to carry the message 
closer to its final destination .The forwarding strategy depends on the energy level and the number of messages to be delivered before encountering the message destination using information on nodes that have already received a copy of the message and the fuzzy logic to decide whether the encountered node should carry the message or should wait to meet one of the nodes that have already received a copy. With this new strategy DTN nodes will be able to save energy due to the operation of transmission and reception of message copies when one of the nodes which have already received a copy have managed to deliver it to its final destination.

The rest of the paper is organized as follows: In Sec.2 we present research methodology and Sec. 3 is devoted to the presentation of energy consumption model for DTN nodes. Sec. 4 defines the proposed routing protocol and the details of implementation. Simulation results are presented and analyzed in Sec.5. Finally, we conclude this paper in Sec.6.

\section{RESEARCH METHODOLOGY}

In this section we describe the Fuzzy logic and ant colony used to build the proposed EERPFAnt.

\section{A. Fuzzy logic}

Human beings are capable of taking decisions, even in the case where knowledge is inaccurate or incomplete decision systems based on fuzzy logic, function similarly to the logic of human reasoning rather than the rigid calculations, using human language to describe input information to reach a conclusion. Indeed, in the fuzzy logic, the digital data are expressed in the form of linguistic variables which can be represented using the membership functions. All results are obtained by combining the linguistic variables with predefined rules that define the relationship between the input and output variables. Then they are converted in crisp data so they can be implemented by the external system.

This paper focuses on the improvement of DTN routing protocol performance by decision making using fuzzy logic.

The fuzzy inference system proposed in this paper receives two input variables and provides a single output variable. The two input variables are the result of the subtraction between the transition probabilities (ETP) nodes encountered (in communication) and the normalized sum of the connectivity degree of nodes that have already received a copy of the message (NSP). The output variable is the decision of the routing protocol (RD).

In this paper we have chosen to use a relatively simple triangular membership functions for each input variable (ETP and NSP) due to their simple implementation and are inexpensive in terms of computation time and memory space.

The input fuzzy sets are three sets; low, medium and high and output are two sets; sending and not sending the message. The basic rule is made up of nine rules that were designed to evaluate the input variables in order to decide on the message transmission. The rules used are shown in Table 2. For example, Rule 1 indicates that if the transition probability is low and the normalized sum of the connectivity degree is low, then the node sent the message to the encountered node. The Fig.1 shows a fuzzy inference system containing the three main steps namely the fuzzification, the fuzzy inference machine and defuzzification.

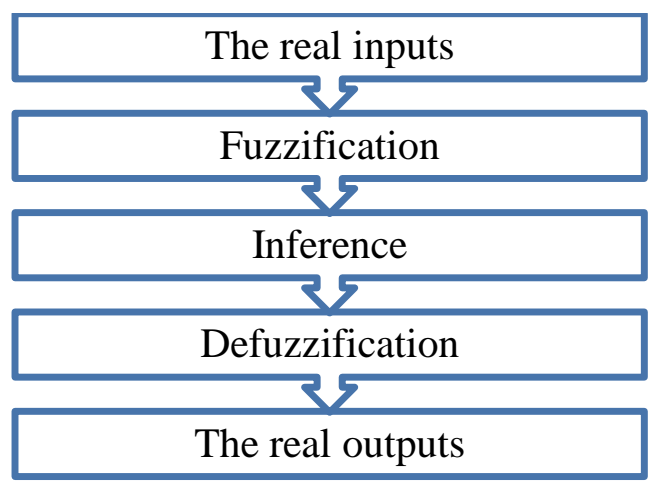

Fig.1. Fuzzy Inference System

\section{- Fuzzification}

Fuzzification is the conversion process of the digital value of the input variables to a fuzzy value using a fuzzy membership function. The fuzzy membership functions of ETP and NSP are defined in the Fig. 4. These membership functions are defined taking into account the following points. First, we must avoid sending the message to a node that has a transition probability very close to the transition probability of the message source node (the gap between the transition probability is low) and has a very high connectivity degree (NSP), with nodes that have already received a copy of the message and have a great chance to meet it before encountering the message destination. However, if the gap between the transition probabilities exceeds a certain level and the value of NSP is less than a certain level then the message must be transmitted to the encountered node.

\section{- The inference}

It is in this stage where the fuzzy rules are established. They link the input and the output variables in order to draw fuzzy deductions. A fuzzy rule comprises a premise like "If ... And if ..." and a fuzzy inference of the type "Then ..." (If X is A And Y is B Then $\mathrm{Z}$ is C). We have chosen to apply the minimum and maximum operations which have the advantage of simplicity in computing for combining the input variables to obtain the output variable.

\section{- Defuzzification}

Defuzzification is the last step to convert the values obtained from the combination of rules applied to input variables into a physical quantity. As there may be several rules at the same time, we use the center of gravity method (COG) to combine the results into a final result. Then it remains for us to make the final decision. 


\section{B. Ant colony}

The observation of the behavior of natural ants showed that the ants in a given colony can find the best way saying shortest between their nest and the food source. The key element of this behavior is the chemical substance called pheromone. In fact, ants lay this substance while moving along the paths traveled in order to inform other insects on the area that has been explored. The path that has received a significant amount of pheromone compared to other paths attracts new ants. Indeed, the shortest path can be provided more rapidly and more frequently visited by ants and will be marked by a greater intensity of pheromone. Foraging behavior of natural ants has been a major inspiration to solve discrete optimization problems in various fields of engineering especially in designing routing protocols.

ACO (ant colony optimization) is the first optimization algorithm based on ant colony that has been proposed to solve the traveling salesman problem [16]. Thereafter; it became a guiding element to researchers for other applications. For routing protocols, the basic idea behind the ACO algorithms is to imitate the behavior of ants by generating artificial ants simultaneously and independently at the DTN nodes to collect information through a collective learning process in order to use it to select the best relay to route messages between the source and the destination. In this paper nodes collect information (energy level, the free space buffer and the most encountered nodes), Then use them to update the tables of pheromone at intermediate nodes for each destination. They in turn, the ants use the pheromones tables to choose the relay that are more likely to carry the messages to their destinations by combining the pheromone values and the heuristic to calculate the transition probability between nodes.

\section{ENERGY CONSUMPTION MODEL FOR DTN NODES}

The lifetime of a DTN node is often defined by the duration of its battery which makes the energy consumption a constraint that must be taken into account when designing a routing protocol in DTN network. Indeed, the main objective of DTN routing protocols [17] is to increase the delivery ratio of message. Therefore, the use of multiple copies of each message increases the amount of energy used for transmission and reception of these copies and consumes more buffer space. However, routing protocols that use fewer copies of the messages will be more energy efficient.

The energy module [18] that we used to simulate the concept of energy consumption by DTN nodes can be described as follows:

- The nodes batteries are discharged according to the operation performed; receiving, transmitting, storing a message or seeking for a neighbor node.

- The amount of energy consumed by each operation is illustrated in Table 1.
- Nodes can recharge the batteries in case of exhaustion.

Table 1. Energy Parameters

\begin{tabular}{|c|c|c|}
\hline Parameter & $\begin{array}{l}\text { Value } \\
\text { (units) }\end{array}$ & Description \\
\hline $\begin{array}{l}\text { Initial } \\
\text { Energy }\end{array}$ & $4,8 \mathrm{j}$ & Battery Capacity :battery full \\
\hline $\begin{array}{c}\text { Scan } \\
\text { Interval }\end{array}$ & $32 \mathrm{~s}$ & $\begin{array}{l}\text { The node switches periodically (32 } \\
\text { seconds) to scan state. }\end{array}$ \\
\hline $\begin{array}{c}\text { Scan } \\
\text { Energy }\end{array}$ & $\begin{array}{c}0.92 \\
\mathrm{~mW} / \mathrm{s}\end{array}$ & $\begin{array}{l}\text { The node spends energy in order to } \\
\text { detect the presence of neighbors. }\end{array}$ \\
\hline $\begin{array}{l}\text { Transmit } \\
\text { Energy }\end{array}$ & $\begin{array}{c}0.08 \\
\mathrm{~mW} / \mathrm{s}\end{array}$ & The node is sending messages. \\
\hline $\begin{array}{l}\text { Scan } \\
\text { Response } \\
\text { Energy } \\
\end{array}$ & $\begin{array}{c}0.1 \\
\mathrm{~mW} / \mathrm{s}\end{array}$ & $\begin{array}{l}\text { The amount of energy consumed in } \\
\text { device discovery response. }\end{array}$ \\
\hline $\begin{array}{l}\text { Receive } \\
\text { Energy }\end{array}$ & $\begin{array}{c}0.08 \\
\mathrm{~mW} / \mathrm{s}\end{array}$ & The node is receiving messages. \\
\hline idle Energy & $\begin{array}{c}0.01 \\
\mathrm{~mW} / \mathrm{s}\end{array}$ & $\begin{array}{l}\text { In this state the node has its network } \\
\text { interface Sleeping but it can be detected } \\
\text { by other nodes. }\end{array}$ \\
\hline
\end{tabular}

Each DTN node has five states:

(1) OFF: the node cannot communicate with the others nodes.

(2) Idle: the Network Node Interface is inactive and can be detected by its neighboring nodes.

(3) Scan: The node tries to find his neighborhood.

(4) Transmission: The node sends messages.

(5) Reception: The node receives messages.

This module controls the energy consumption depending on the state of the node, in fact, the energy required for the node when it is idle is zero, the energy spent by a node in the case of transmission or reception depends on the operation duration, it is equal to the result of multiplying the total duration of the operation and the predefined amount of energy expended per unit of time that is provided as a parameter for each node class in the configuration of the simulator. The same approach is used in the case of research neighboring nodes. So that charging of the node battery is close to reality, the module allows each node to carry out the charging of its battery at time intervals selected in a random manner in order to avoid charging of all the batteries at a time or all at very regular intervals.

\section{PROPOSED APPROACH}

The main objective of our routing protocol is to deliver the maximum messages to their final destinations without wasting energy consumed by nodes. For this, the nodes exchange messages based on two main pieces of information namely: the transition probability between encountered nodes and the message destination and the transition probability between encountered node and nodes that have already received a message copy. This strategy allows the nodes to avoid sending messages to other nodes when they will encounter the relays that have already received a copy before encountering message destination. 
In this section, we present our strategy named EERPFAnt (Energy Efficient Routing Protocol based on Fuzzy Ant colonies). It is inspired by ant colony to seek the best relays; those offering a greater chance that the message reaches its final destination and that will have sufficient energy to deliver it. EERPFAnt uses fuzzy logic to balance the load between the nodes in order to solve the problem of network resources consumption; energy and buffer space.

In this approach the nodes take into account the energy level of the other nodes at the time of contact and messages that will be delivered before encountering the desired destination to estimate the energy of the nodes at the time of meeting with the desired destination in order to avoid the participation of some nodes to route messages even if they will not have sufficient energy to bring them closer to the final destination. The overall objective of our approach is to deliver messages from the source to the destination through the relay nodes. To do this, each node has to choose among its neighbors one that has great chance to bring the message closest to its final destination. This is done by calculating the transition probability between the neighbor's node and the message destination.

\section{A. The formal description}

The detailed description of EERPFAnt algorithm is given by the following two phases:

\section{1) First phase: Collecting information}

In this phase, each node set up and maintains a list of connectivity degree with each encountered node, exchanges information about the energy level of the battery, the buffer space available and the set of the most encountered nodes in order to update the tables indicated in the Fig.2: TableEn, TableBfr and TableHops. The information collected will be used in the second phase of the algorithm to search the relays that have more chance to meet the final destination of the message.

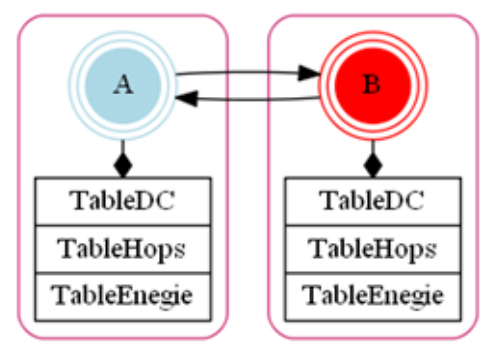

Fig.2. The information exchange between the nodes A and B

The degrees of direct and indirect connectivity used by each node are recalculated at each opportunistic encounter using (1), (2), (3) and (4). Indeed at every encounter between two nodes $a$ and $b$, both nodes update their:

- $\quad$ Direct connectivity table (TableDC) :( $\left.\Gamma^{\mathrm{d}}, \mathrm{t}\right)$; each node reinforces the direct connectivity degree, $\Gamma^{\mathrm{d}}$ $(\mathrm{a}, \mathrm{b})$, with the node encountered using (1) and reduces that of the other nodes using (2).

$$
\begin{gathered}
\Gamma_{t_{i}}^{d}(a, b)=\Gamma_{t_{i-1}^{d}}^{d}(a, b)+P_{0}\left(1-\Gamma_{t_{i-1}}^{d}(a, b)\right) \exp \left(-\left(t_{i}-t_{i-1}\right)\right) \\
\Gamma_{t_{i}}^{d}(a, b)=\Gamma_{t_{i-1}}^{d}(a, b)-\left(1-P_{0}\right)\left(1-\Gamma_{t_{i-1}^{d}}^{d}(a, b)\right) \exp \left(-\left(t_{i}-t_{i-1}\right)\right)
\end{gathered}
$$

- Indirect connectivity table (TableIC), each node uses direct connectivity table (TableDC) of the other node to reinforce the degree of indirect connectivity $\Gamma^{\text {id }}(\mathrm{a}, \mathrm{c})$ at time $\mathrm{t}$, with each known destination c using (3) and reduces the indirect connectivity degree with the other nodes using (4).

$$
\begin{aligned}
& \left.\Gamma_{t_{i}}^{i d}(a, c)=\Gamma_{t_{i-1}}^{i d}(a, c)+\Gamma_{t_{i-1}}^{d}(a, b) \Gamma_{t_{i-1}}^{d}(b, c)\right) \exp \left(-\left(t_{i}-t_{i-1}\right)\right) \\
& \Gamma_{t_{i}}^{i d}(a, c)=\Gamma_{t_{i-1}}^{i d}(a, c)-\left(1-\Gamma_{t_{i-1}}^{i d}(a, c)\right) \exp \left(-\left(t_{i}-t_{i-1}\right)\right)
\end{aligned}
$$

Where $\Gamma^{\mathrm{d}}(\mathrm{a}, \mathrm{b})$ and $\Gamma^{\mathrm{id}}(\mathrm{a}, \mathrm{b})$ are, respectively, the direct and the indirect connectivity degree between the nodes $\mathrm{a}$ and $\mathrm{b}, \mathrm{P}_{0} \in[0,1]$ is the update factor, $\mathrm{t}_{\mathrm{i}}$ is the updating time of the connectivity degree. If the node has not stored a value for a destination $b$, the connectivity degree $\Gamma^{\mathrm{d}}(\mathrm{a}, \mathrm{b})$ or $\Gamma^{\mathrm{id}}(\mathrm{a}, \mathrm{b})$ is assumed to be zero.

After updating the connectivity degrees both nodes a and $b$ exchange information on the available buffer space $\left(B_{S}\right)$, battery level $\left(E_{1}\right)$, the list of sets composed of three node that have a high connectivity degree with each known destination ( $\{\mathrm{N} 1, \mathrm{~N} 2, \mathrm{~N} 3\})$. The information collected will be used to update the table: TableHops $<\mathrm{Ni}, \mathrm{t},\{\mathrm{Ni} 1, \mathrm{Ni} 2, \mathrm{Ni} 3\}>$ the three nodes that have the highest direct connectivity degree with the destination $\mathrm{Ni}$ et $\mathrm{t}$; the time of recording, the table TableEnegie $<\mathrm{N}, \mathrm{t}, \mathrm{B}_{\mathrm{s}}, \mathrm{E}_{\mathrm{l}}>$; the battery level at the time of recording and the buffer space.

\section{2) Second phase: search hops}

In this phase, each node seeks among its neighbors based on the transition probability (11), the node that has more chance to approach the message to its final destination, with sufficient energy so that can deliver it. The transition probability is calculated by combining the amount of pheromone and visibility between nodes.

a) Le calcule de la visibilité $\zeta_{i j}$.

\section{- $\quad$ The predecessors and the Successors}

The construction of the predecessor list of node $\mathrm{N}, \mathrm{R}_{1}$ $(\mathrm{N})=\{\mathrm{Ni} / \in \mathrm{N}$ TableHops $\mathrm{Ni}\}$, comes down to search for the node $\mathrm{N}$ in column Set $(\mathrm{Ni})$ of the table TableHops $\langle\mathrm{Ni}, \mathrm{t},\{\mathrm{Ni} 1, \mathrm{Ni} 2, \mathrm{Ni} 3\}\rangle$, then if the node $\mathrm{N}$ is among the set $\{\mathrm{Ni} 1, \mathrm{Ni} 2, \mathrm{Ni} 3\}$, the node $\mathrm{Ni}$ is added to the list $\mathrm{R} 1$ $(\mathrm{N})$. To create the list $\mathrm{R} 2(\mathrm{~N})$, we search in the same manner all predecessors of all nodes in the list R1 (N). To construct of the list $\mathrm{F}_{1}(\mathrm{~N})=\{\mathrm{Ni} / \mathrm{N} \in$ TableHops:Set(Ni)\}, we seek the node $\mathrm{N}$ in the column $\mathrm{Ni}$ of the table TableHops $\langle\mathrm{Ni}, \mathrm{t},\{\mathrm{Ni} 1, \mathrm{Ni} 2, \mathrm{Ni} 3\}\rangle$, then 
if it exists the set $\{\mathrm{Ni} 1, \mathrm{Ni} 2, \mathrm{Ni} 3\}$ is added to the list $\mathrm{F}_{1}$ $(\mathrm{N})$. To create the list $\mathrm{F}_{2}(\mathrm{~N})$, we search in the same manner all successors of all nodes in the list $\mathrm{F}_{1}(\mathrm{~N})$.

In general, as shown in Fig.3, the construction of the list Fi $(\mathrm{N})$ depends on the list Fi-1 $(\mathrm{N})$, the nodes of level $\mathrm{Fi}$ have as predecessors nodes of the level $\mathrm{Fi}_{-1}(\mathrm{D})$ and as successor nodes of the level $\mathrm{Fi}_{+1}$. In this paper the construction process of levels continues until the third level F3 (N) for the successors and only the first level R1 (N) for the predecessors of the desired destination as shown in Fig.3 . Ri (N) is the predecessor list of node $\mathrm{N}$ at $\mathrm{i}$ hops; the set of nodes that consider $\mathrm{N}$ as their best successor at i hops, Fi (N) is the successors set of node $\mathrm{N}$ at $\mathrm{i}$ hops; the set of nodes at $\mathrm{i}$ hops that $\mathrm{N}$ considers them as the best predecessors.

\section{b) The calculation of the encounter cost}

After creating the set of nodes at one hop, containing the nodes that have a high probability of encountering the message destination, we calculate the heuristics Cost (D) (5) and Cost (R1 (D)) (6) that determine the membership degree of the successors of a given node $\mathrm{A}$ to the set of the predecessors of the destination $D$. With $\beta \mathrm{j}=1$ if $\mathrm{N} \in$ $\mathrm{Fj}(\mathrm{D}), \beta \mathrm{j}=0$ if $\mathrm{N} \notin \mathrm{Fj}(\mathrm{D})$.

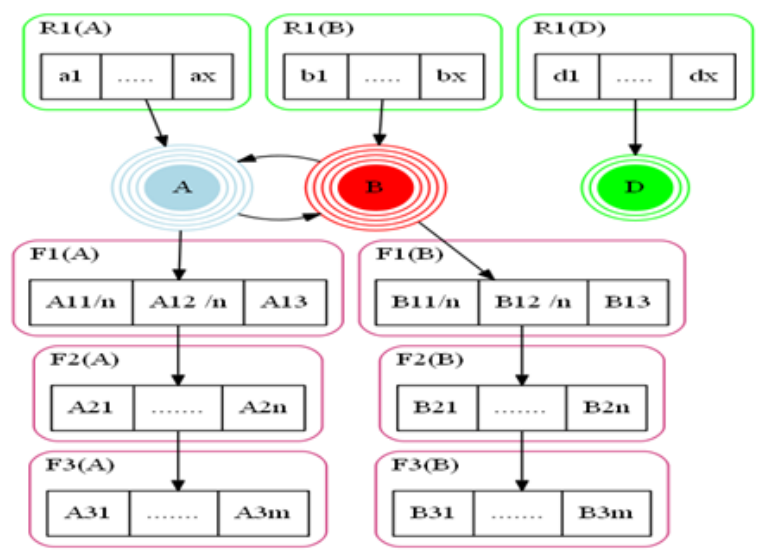

Fig.3. The predecessors and successors

$$
\begin{gathered}
\operatorname{Cost}(D)=\sum_{i=1}^{n} \frac{f_{i}(D)}{\operatorname{card}\left(F_{i}(A)\right)} \beta_{i} \alpha^{i} \\
\operatorname{Cost}\left(R_{1}(D)\right)=\sum_{N \in R_{1}(D)} \sum_{i=1}^{n} \frac{f_{i}(N)}{\operatorname{card}\left(F_{i}(A)\right)} \beta_{i} \alpha^{i} \\
\operatorname{Cost}(\mathrm{S}, \mathrm{D})=\operatorname{Cost}(\mathrm{D})+\operatorname{Cost}\left(\mathrm{R}_{1}(\mathrm{D})\right) \\
\mathrm{E}_{1}(\mathrm{~A} \rightarrow \mathrm{D})=\mathrm{E}_{1}-\sum \varepsilon \mathrm{N}_{\mathrm{mgg}}\left(\mathrm{A} \rightarrow \mathrm{N}_{\mathrm{i}}\right) \Gamma_{\mathrm{d}}\left(\mathrm{A}, \mathrm{N}_{\mathrm{i}}\right)
\end{gathered}
$$

The encounter cost is determined by the combination of the heuristics Cost (D) and Cost ( $R_{1}$ (D)) using (7). With $F_{i}(N)$ is the membership degree of the node $N$ to the Set $F_{i}(A)$. Card $\left(F_{i}(R)\right)$ is the number of elements of the Set $F_{i}(D) . \alpha \in[0,1]$ is the update factor.

\section{c) The estimation of energy level}

The approach used in this paper allows to estimate the amount of energy at the time of encounter with the desired destination; node $\mathrm{D}$, in fact, the nodes use the of direct connectivity degree to determine the messages that will be transmitted before encountering destination $\mathrm{D}$. When a node A wants to estimate its energy level at the time of encounter with a node $\mathrm{D}$, It relies on the direct connectivity degree to determine the nodes $\mathrm{Ni}$; the destinations of the messages stored in the buffer of the node $A$ which have a connectivity degree $\Gamma^{\mathrm{d}}\left(\mathrm{A}, \mathrm{N}_{\mathrm{i}}\right)$ greater than that of the destination $\mathrm{D} ; \Gamma^{\mathrm{d}}(\mathrm{A}, \mathrm{D})$.Eq. (8) gives us an estimate of the energy by the combination of the selected messages number, the connectivity degree of each node $\mathrm{N}_{\mathrm{i}}$ and the current energy level of the node $\mathrm{A}$.

With: $N_{\text {msg }}\left(A \rightarrow N_{i}\right)=\{$ the number of messages stored in the buffer of the node $\mathrm{A}$ and have as destination $\mathrm{Ni}$ node that satisfies the condition $\Gamma^{\mathrm{d}}(\mathrm{A}, \mathrm{Ni})>\Gamma^{\mathrm{d}}$ (A ,D) $\}. \varepsilon$ is the amount of energy needed to transmit a given message and $E_{l}(A \rightarrow D)$ is the energy estimate at the time of encounter of node $\mathrm{A}$ with the destination $\mathrm{D}$.

\section{d) The visibility between nodes $\zeta_{A D}$}

Visibility between nodes takes into account the energy level estimated at the time of the encountering node $\mathrm{D}$ by the node $\mathrm{A}$ and the encounter cost of neighbors nodes at one hop of the destination $D$ with A neighbors nodes. Eq. (9) determines the visibility between nodes A and $\mathrm{D}$, with $\beta$ is a parameter that controls the relative importance between the estimated energy and the cost encounter.

$$
\zeta_{\mathrm{AD}}=\beta \mathrm{E}_{1}(\mathrm{~A} \rightarrow \mathrm{D})+(1-\beta)(\operatorname{Cost}(\mathrm{A}, \mathrm{D})
$$

\section{e) The amount of pheromone $\eta_{A D}$}

The amount of pheromone $\eta_{\mathrm{AD}}$ deposited by ants between nodes is calculated by combining the direct and indirect connectivity degrees between a given node $\mathrm{A}$ and the desired destination D using (10). Reinforcement and evaporation of the pheromone is done respectively by (1) (3) and (2) (4).

$$
h_{A D}=b G^{d}(A, D)+(1-b) G^{i d}(A, D)
$$

\section{f) The transition probability}

The selection of the relays that have a great chance to meet the final destination of a given message is done based on the transition probability using (11). The calculation of the transition probability takes into account the energy level of encountered nodes, the messages stored in their buffer and the connectivity degree between nodes. With $\mathrm{N}_{\mathrm{ik}}$ is in our approach, the set of neighboring nodes, of the node that carries the message. With $\beta$ is a parameter that controls the relative importance between the amount of pheromone and visibility between nodes. 
$\left\{\begin{array}{l}p_{i j}^{k}(t)=\frac{\alpha \zeta_{i j}(t)+(1-\alpha) \eta_{i j}}{\sum_{l \in N_{i}^{k}(t)} \alpha \zeta_{i l}(t)+(1-\alpha) \eta_{i l}} j \in N_{i}^{k} \\ 0 \quad \text { otherwise }\end{array}\right.$

\section{g) The decision of the message transmission.}

\section{(1) Description of Fuzzy Inference System}

In this paper, we use fuzzy logic to determine if the node must participate to route a message or whether it should wait for another opportunity. The objective of this strategy is to allow nodes to avoid sending the message to nodes that have a chance to meet one of the nodes that have already received a copy of message.

The inputs variables to fuzzy controller are:

- $\quad$ ETP: The gap between the transition probabilities which is calculated using (13).

- NSP: The normalized sum of the connectivity degree between the encountered node and all nodes that have already received a copy of the message. This variable is calculated using (14).

The output variable of the fuzzy controller System is the routing decision (DR); the node transmits the message or waits to another opportunity.

The calculation steps of the Fuzzy cost are elaborated as follows:

\section{(2) Fuzzification}

The two input variables are: The gap between the transition probability (EPT) and the normalized sum of connectivity degree with the relays that have already received a copy (NSP). The terms low, medium and high are used to describe EPT and NSP. The membership functions that we have used are triangular in type due to their simplicity and computational efficiency.

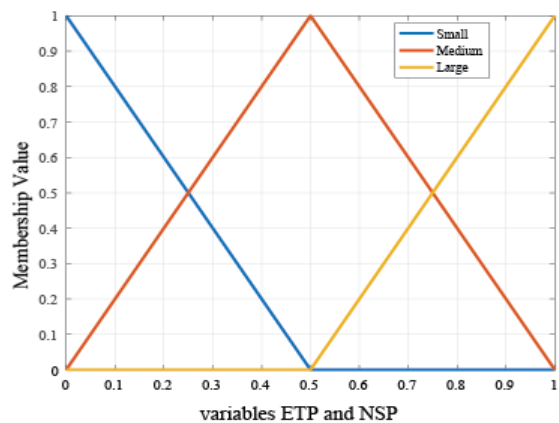

Fig.4. Fuzzy Membership function of the ETP and NSP

Fig.4 and Fig.5 represent the inputs and the output variables respectively. The output variable is a fuzzy cost which determines whether a node must participate to route the message or wait for an opportunity to meet with another relay, which carries a copy of the message, before encountering the message destination or another node that has a greater chance of meeting the message destination with enough energy. The fuzzy costs are used to decide whether to send the message or wait for another opportunity.

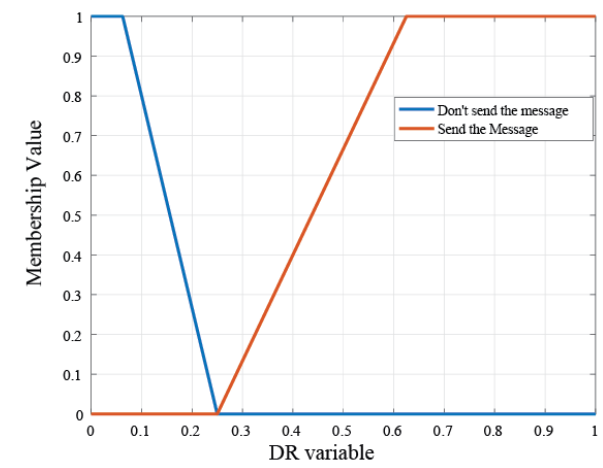

Fig.5. Fuzzy Membership functions for the output (DR)

\section{(3) Inference}

In this step our system uses fuzzy rules of the form (If...Then) and the inputs will then be combined with the AND operator. Table 2 shows the fuzzy rules for the decision of the routing protocol.

Table 2 . The fuzzy rules

\begin{tabular}{cccc}
\hline If & \multirow{2}{*}{ And } & If NSP & Then \\
EPT & Small & RD \\
Small & Medium & Send Message \\
Small & large & Don't Send Message \\
Small & Small & Don't Send Message \\
Medium & Medium & Send Message \\
Medium & large & Send Message \\
Medium & Small & Don't Send Message \\
large & Medium & Send Message \\
large & large & Send Message \\
large & & Don't Send Message \\
\hline
\end{tabular}

\section{(4) Defuzzification}

In this step we use the property of gravity center by using (12) to determine the value of the output variable by combining the input variables and membership degree. Where FC is the fuzzy cost, $\mathrm{xi}$ is the element and $\mu\left(\mathrm{x}_{\mathrm{i}}\right)$ is the membership function.

$$
F C=\frac{\sum_{\text {Allrules }} x_{i} \mu\left(x_{i}\right)}{\sum_{\text {Allrules }} \mu\left(x_{i}\right)}
$$

In this phase we calculate:

- $\quad$ The gap of the transition probability (ETP).

$$
\mathrm{ETP}=\mathrm{P}(\mathrm{A}, \mathrm{D})-\mathrm{P}(\mathrm{S}, \mathrm{D})
$$

- The normalized sum of the connectivity degree.

$$
N S P=\frac{\sum_{\operatorname{set}\left(N_{i}\right)} \eta_{\mathrm{AN}_{i}}}{\sum_{\operatorname{set}\left(N_{j}\right)} \eta_{\mathrm{AN} j}}
$$


Where NSP is the normalized sum of the transition probabilities, Set $(\mathrm{Ni})$ is the set of nodes that have already received a copy of the message with the proviso that $\eta_{\mathrm{ANi}}$ is greater than $\eta_{\mathrm{AD}}$ and $\mathrm{Set}(\mathrm{Nj})$ is the set of all nodes that have already received a copy of the message.

Both variables ETP and NSP will play the role of input variables of the fuzzy inference system that we have presented in Sec.2, to decide whether the message should be sent to the node $\mathrm{A}$ or the node $\mathrm{A}$ waits until it encounters one of the nodes of the set Set $(\mathrm{Ni})$.

\section{SimULATION AND RESULTS}

\section{A. Simulation Environment}

The ONE [20-22] simulator (opportunistic Network Environment Simulator) is used to evaluate the performance of the proposed approach compared to the routing protocols Epidemic, Spray and Wait and ProPHET. The nodes used in the simulations are of two types; there is a pedestrian (50) and a vehicle (6), which can generate and receive messages. These two types of nodes move with different mobility in the simulation region. For pedestrians, the speed is $0.5 \mathrm{~m} / \mathrm{s}$ and $1.5 \mathrm{~m} / \mathrm{s}$. The vehicle speed is in the range $(6 \mathrm{~m} / \mathrm{s} \sim 12 \mathrm{~m} / \mathrm{s})$. The simulation parameters are shown in Table 3.

Table 3. Simulation Parameters

\begin{tabular}{|l|l|}
\hline Simulation Parameters & Simulation values \\
\hline Simulation time & $10000 \mathrm{~s}-80000 \mathrm{~s}$ \\
\hline Transmission range & $10 \mathrm{~m}$ \\
\hline Packet transmission speed & $250 \mathrm{Kbyte} / \mathrm{s}$ \\
\hline Updating factors of EERPFAnt : $\alpha$ and $\beta$ & 0.75 \\
\hline Updating factor of PROPHET & 0.75 \\
\hline Aging constant of PROPHET & 0.98 \\
\hline Transitivity constant of PROPHET & $0: 25$ \\
\hline Initial copies number of Spray and Wait & 10 \\
\hline Buffer size & $50 \mathrm{M}$ \\
\hline Note: P: Pedestrian v: Vehicle \\
\hline
\end{tabular}

\section{B. Simulation Results}

To evaluate the performances of the routing protocol EERPFAnt compared to routing protocols Epidemic, Spray and Wait and ProPHET, several simulations were performed with the ONE simulator designed primarily for DTN networks. The simulation results represented in the figures below are the average of 10 simulations for each routing.

Fig.6, represents the delivery ratio by varying the simulation duration, it shows that, the Epidemic Routing has the lowest delivery ratio compared to the other routing protocols, this is due to the blind strategy adopted by this protocol, which influence on the buffer capacity, indeed, the sending of multi copies of each message to all encountered nodes, quickly causes the buffer saturation, hence, nodes drop some messages to make room for others, thereby reducing the delivery ratio thereafter. For the other that are based on the information collected from the network; ProPHET and EERPFAnt have a better delivery ratio than spray and wait, which can be explained by the fact that for a long simulation times, nodes collect more information which helps them to make a good decision to routing their messages, hence a better delivery rate.

Fig.7 shows clearly that the overhead generated by the routing protocol EERPFANT is better than that generated by the other two routing protocols; this is due to the strategy adopted by EERPFAnt that allows nodes to wait to meet other nodes that already have a copy of the message, which minimizes the number of messages transmitted and thereafter improves the generated overhead.

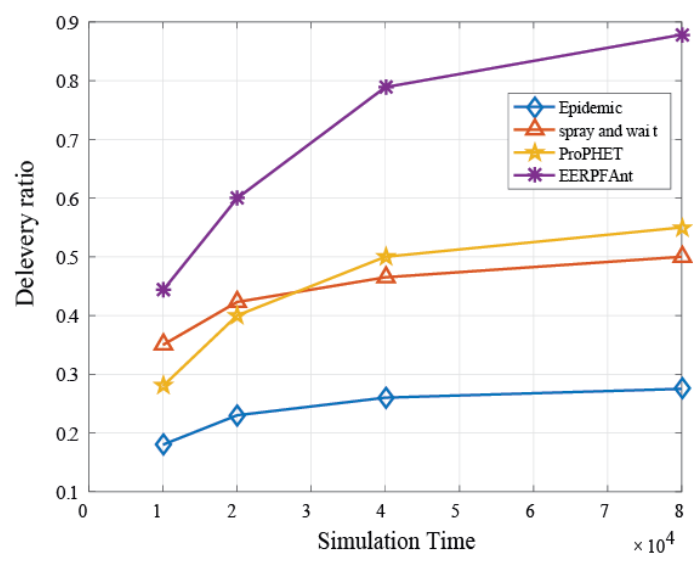

Fig.6. Delivery Ratio w.r.t. Time

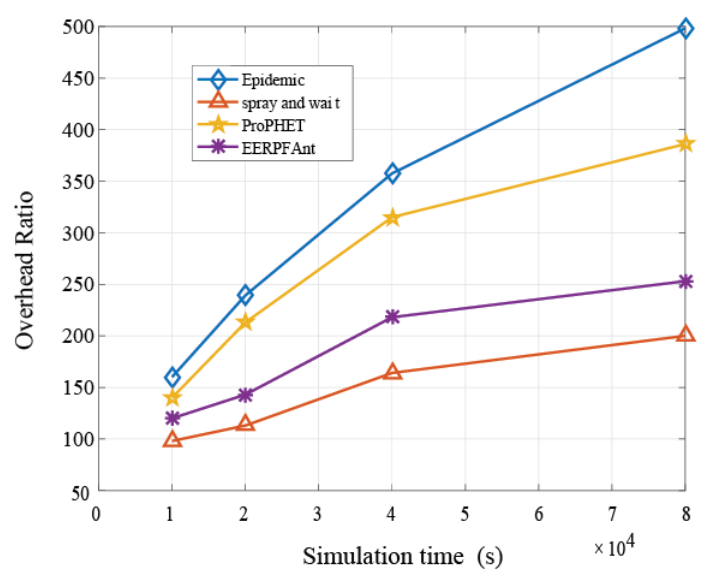

Fig.7. Overhead Ratio w.r.t. Time

Fig. 8 clearly shows that the average residual energy of the nodes decreases with the increase in the number of nodes in the simulation scenario. In fact the more important the number of the nodes is in the simulation scenario, the more exchanges of messages there are between nodes which causes a consumption of more energy. The average remaining energy of nodes that implement one of the routing protocols Spray and Wait and EERPFAnt is higher compared to that of the nodes that implement the routing protocol ProPHET and Epidemic. This is due to the fact that the protocol Spray and Wait uses a limited number of copies and the routing 
protocol EERPFANT forces them to wait to meet nodes that have already received a copy of the message to ensure that they have delivered the message to its final destination.

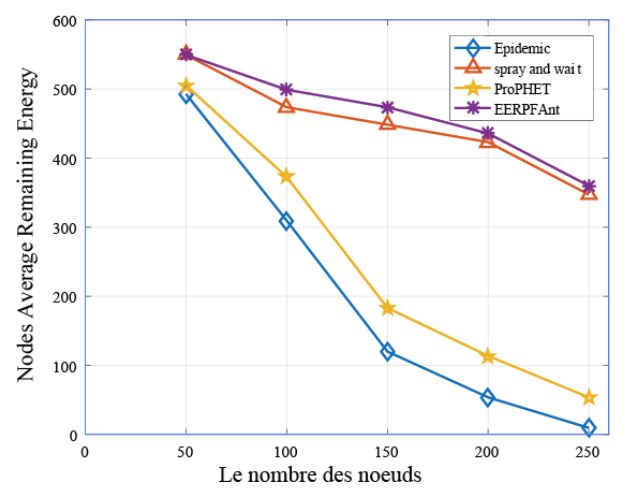

Fig.8. Nodes Average Remaining Energy w.r.t. number of Nodes

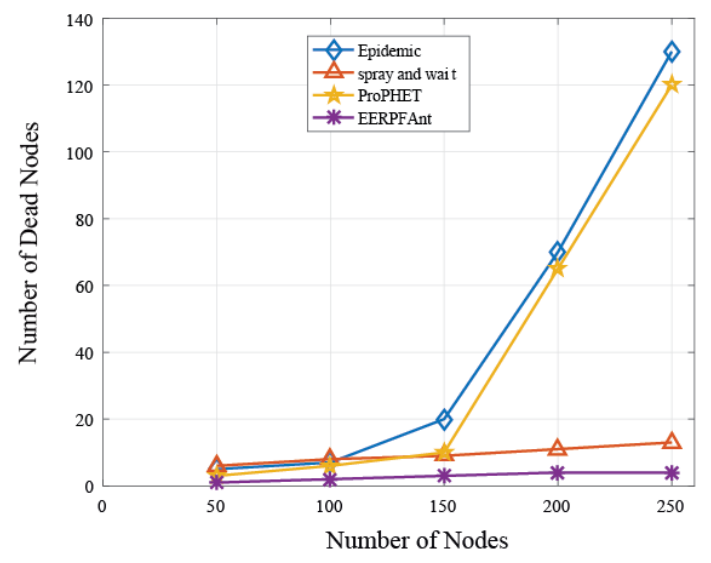

Fig.9. Number of Dead Nodes w.r.t. number of Nodes

In Fig.9, we observe that increasing the number of nodes that implement the routing protocols; Epidemic and ProPHET, leads to an increase in the number of nodes that will be depleted energetically. However the routing protocols Spray and Wait and EERPFAnt have improved the life of the nodes, as they use the information of the energy level of nodes and the information on the nodes that have already received a copy of message.

\section{CONCLUSIONS}

In this paper, we presented a new intelligent and efficient routing protocol named EERPFAnt for DTN network, inspired by the ant colony intelligence, to find the best path between nodes and enhanced by the fuzzy logic system for decision making. As mobility, energy and buffer of nodes are the main challenges in designing the routing protocol. The proposed algorithm exploits encounters history between nodes to calculate the amount of pheromone deposited among them, taking into account the remaining energy level of nodes and messages stored in their buffer to determine the visibility between nodes. The information about the nodes that have already received a copy of the message is used to determine whether the relays are able to participate or not in the message routing.

We tried to find a balance between high message delivery ratio and efficient use of network resources. Indeed, our approach considers the residual energy of the nodes at the time of the encounter with the desired destination and includes this parameter in the calculation of the visibility between nodes, in order to calculate the transition probability between the relay nodes and the searched destination, based on the principle of ant colony optimization, to select the nodes that have a great chance to meet the message destination with sufficient energy so as to be able to deliver it.

To minimize the number of message copies, we use fuzzy logic for taking a decision to forward the message to a given node, or wait to meet one of the relays that have already received a copy of this message which allows conserving energy of transmission-reception due to the message diffusion, while one of its copies is successfully delivered to its destination.

The simulation results showed that the EERPFANT routing protocol presented in this paper has provided best delivery ratio with minimal overhead and efficiently manages the battery of the nodes, compared to other routing protocols DTN widely recognized; Epidemic, spray and wait and ProPHET.

\section{REFERENCES}

[1] Fall, Kevin. "A delay-tolerant network architecture for challenged internets," Proceedings of the conference on Applications, technologies, architectures, and protocols for computer communications. ACM, 2003.

[2] Cerf, Vinton, et al. "Delay-tolerant networking architecture," $\mathrm{N}^{\circ}$. RFC 4838, 2007.

[3] Fall, K., Farrell, S., "DTN: an architectural retrospective," Selected Areas in Communications, IEEE Journal on, vol.26, $\mathrm{N}^{\circ} .5$, pp.828-836, June 2008.

[4] Safdar, Muhammad, Khan, Izaz Ahmad, Ullah, Farman, et al. "Comparative Study of Routing Protocols in Mobile Adhoc Networks," International Journal of Computer Science Trends and Technology, Vol.4, $\mathrm{N}^{\circ} 2$, p. 2347 8578, 2016.

[5] Eshghi, Soheil, Khouzani, M. H. R., Sarkar, Saswati, et al. "Optimal energy-aware epidemic routing in DTNs," IEEE Transactions on Automatic Control, vol. 60, $\mathrm{N}^{\circ} 6$, p. 1554-1569, 2015.

[6] Choksatid, Teerapong et Prabhavat, Sumet. "An epidemic routing with low message exchange overhead for delay tolerant networks," In: Progress in Systems Engineering. Springer International Publishing, p. 429-436, 2015

[7] Hernandez, Roberto, Cesar Cardenas, and David Munoz. "Epidemic routing in vehicular delay-tolerant networks: The use of heterogeneous conditions to increase packet delivery ratio," Smart Cities Conference (ISC2), IEEE First International. IEEE, 2015.

[8] Derakhshanfard, Nahideh, Sabaei, Masoud, et Rahmani, Amir Masoud. "Sharing spray and wait routing algorithm in opportunistic networks," Wireless Networks, vol. 22, N 7, p. 2403-2414,2016.

[9] WANG, En, YANG, Yongjian, WU, Jie, et al. "A buffer management strategy on spray and wait routing protocol in DTNs," In: Parallel Processing (ICPP), 2015 44th International Conference on. IEEE, p. 799-808, 2015. 
[10] LEE, Ho-Jong, NAM, Jae-Choong, SEO, Won-Kyeong, et al. "Enhanced PRoPHET routing protocol that considers contact duration in DTNs," In: Information Networking (ICOIN), International Conference on. IEEE, p. 523-524, 2015.

[11] HAN, Seung Deok et CHUNG, Yun Won. "An improved PRoPHET routing protocol in delay tolerant network," The Scientific World Journal Vol. 2015, Article ID 623090, 2015.

[12] M.Ababou, R. Elkouch, M. Bellafkih and N.Ababou, "New Strategy to optimize the Performance of Spray and wait Routing Protocol," International journal of Mobile Network Communications \& Telematics (IJMNCT), Vol. 6, No. 2, 2014.

[13] Ababou, R. Elkouch, M. Bellafkih and N.Ababou, "Impact of Mobility Models on Supp-Tran Optimized DTN Spray and wait routing protocol," International Journal of Wireless \& Mobile Networks(IJWMN), Vol. 4, No. 2, 2014.

[14] Susilawati, Anita, TAN, John, Bell, David, et al. "Fuzzy logic based method to measure degree of lean activity in manufacturing industry," Journal of Manufacturing Systems, vol. 34, p. 1-11, 2015.

[15] Sahana, Sudip Kumar, Mohammad, AL-Fayoumi, et Mahanti, Prabhat Kumar. "Application of Modified Ant Colony Optimization (MACO) for Multicast Routing Problem," International Journal of Intelligent Systems \& Applications (IJISA), vol. 8, no 4, 2016.

[16] Akhand, M. A. H., Shill, Pintu Chnadra, HOSSAIN, Md Forhad, et al. "Producer-Scrounger Method to Solve Traveling Salesman Problem," International Journal of Intelligent Systems and Applications (IJISA), vol. 7, no 3, p. 29, 2015.

[17] Abdekader, Tamer, Naik, Kshirasagar, Nayak, Amiya, et al. "A performance comparison of delay-tolerant network routing protocols," IEEE Network, vol. 30, Nº 2, p. 46-53, 2016.

[18] Silva, Denis Rodrigues, Costa, António, et Macedo, Joaquim. Energy impact analysis on DTN routing protocols. ExtremeCom , p. 1-6, 2012.

[19] Ari Keränen, Jörg Ott and Teemu Kärkkäinen, "The ONE Simulator for DTN Protocol Evaluation, "available at netlab.tkk.fi/tutkimus/DTN/theone," 2009.

[20] LIU, $\mathrm{Xu}$ et CHEN and Yuanzhu. "Report of A DTN Simulator-THE ONE," 2013.

[21] Harminder Singh Bindra,A L Sangal,"Need of Removing Delivered Message Replica from Delay Tolerant Network - A Problem Definition," IJCNIS, vol.4, no.12, pp.59-64, 2012.

\section{Authors' Profiles}

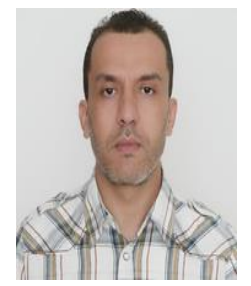

Mr. Mohamed Ababou received his Ph.D degree from National Institute of Posts and Telecommunications Rabat, Morocco. He received his Master Course in Informatics Telecommunications and Multimedia from Faculty of Science, Mohammed V University, Rabat, Morocco. His current research focuses on computer science and

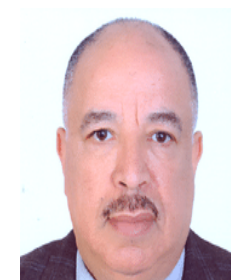

Rachid EL Kouch is a Professor at the National Institute of Post and Telecommunications (INPT) Rabat Morocco. $\mathrm{He}$ is the Manager of PABX Laboratory, attached to Systems and Communications Department. Since 1981, he started his carrier as a telecommunications engineer, and then in 1989 he got his Masters degree of Science in Telecommunications from the University of Colorado-Boulder in the USA. In 2005, he obtained his doctorate degree in applied mathematical from Mohammed Ben Abdellah UniversityFaculty of Sciences and Techniques of Fez-USMBA. His research fields include mobile delay-tolerant networks, wireless communications.

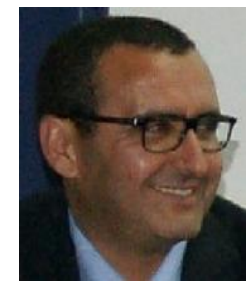

Mostafa Bellafkih received his Ph.D degree from Pierre and Marie Curie University (Paris 6), in 1994. He is also received the Ph.D degree from the Mohammadia School of Engineers, University of Mohamed $\mathrm{V}$ morocco, in 2001.His research interest is in Network Management, Services and QoS, Software Engineering, Computer Communications (Networks), Information Systems (Business Informatics).

How to cite this paper: Mohamed Ababou, Mostafa Bellafkih, Rachid El kouch, " Energy Efficient Routing Protocol for Delay Tolerant Network Based on Fuzzy Logic and Ant Colony", International Journal of Intelligent Systems and Applications(IJISA), Vol.10, No.1, pp.69-77, 2018. DOI: 10.5815/ijisa.2018.01.08

DTN Network. 